\title{
A Wireless Network Firefighter Positioning and Monitoring System and Data Transmission Method Based on Thin AP Framework
}

\author{
Fan Yufeng ${ }^{1}$ Du Yang ${ }^{2}$ and Zhang Lei ${ }^{3}$
}

Shenyang Fire Research Institute of Ministry of Public Security Shenyang 110034

Keywords: Firefighting; Firefighting and Rescue; Firefighter positioning and monitoring system

\begin{abstract}
In this paper, starting from the actual needs of firefighters positioning of firefighting and rescue and the current status of WLAN development in buildings, a wireless network firefighter positioning and monitoring system and data transmission method based on thin AP framework is proposed. The composition, function, data communication protocol and data transmission method of the system are elaborated in detail.
\end{abstract}

\section{Introduction}

With the advancement of wireless network communication technologies, WLAN (Wireless Local Area Networks) has entered an era of rapid development. In most cities in China, offices, shopping malls, hotels, restaurants, large-scale conference venues and even large-scale commercial streets all have achieved full coverage of WIFI signals, providing people with a lot of convenience. The reason is that, on the one hand, wireless communication technology has become more mature and can save manpower and financial resources compared to traditional wired deployment; on the other hand, wireless communication technology can be used as a selling point by companies or places to increase the competitiveness of enterprises and win more customers' favor.

In recent years, with the increasing number of high-rises, super high-rises and large-scale integrated entertainment venues in cities, the fire risk factor has also been increasing. The life and property losses caused by fires in such venues are also rising year by year. In order to cope with this problem, the public security fire department of our country has invested a large amount of manpower and material resources every year to monitor and inspect the integrity of fire protection facilities, and to apply scientific and technological means to achieve real-time monitoring of fire insurance, in order to minimize the fire risk factor in such sites. Once a fire occurs in such a place, how to quickly rescue the internally trapped people and quickly extinguish the fire on the premise of guaranteeing the safety of firefighters is a major problem facing the current firefighting forces.

Based on the above, this paper introduces a wireless network firefighter positioning and monitoring system and data transmission method based on thin AP framework, which is used to solve the technical problems of firefighters' positioning protection in rescue operations during fire fighting.

\section{Wireless Network Firefighter Positioning and Monitoring System Technical Scheme based on Thin AP Framework}

Wireless network firefighter positioning and monitoring system technical scheme.

In workplace, shopping malls, hotels, restaurants, large conferences and other places, the deployment of WLAN is mostly based on thin AP framework. Access Point can also be called wireless bridge or wireless gateway. The so-called thin AP is an AP node that must be managed, debugged and controlled by Wireless Access Point Controller. It cannot work independently and can only work properly with AC. In contrast, fat AP refers to Wireless Router. In addition to wireless access function, it generally has WAN and LAN interfaces, which supports DHCP server, DNS and MAC address cloning, as well as VPN access and firewall security functions. It is mainly used for small places or home Internet access. From the definitions of fat and thin APs, it can be seen that thin AP network deployment mode is the main mode adopted for WLAN networking of large places. The overall network framework has the characteristics of flexible deployment, large network capacity and convenient configuration. 
However, when there is a fire in buildings or other places, the external power supply should be disconnected due to security considerations. In this case, WLAN will lose its network coverage function due to no power supply and internal personnel will not be able to use WLAN either. In WLAN based on thin AP framework, AP power supply adopts POE power supply mode. POE (Power Over Ethernet) refers to the technology which can provide DC power supply for IP-based terminals (IP telephones, wireless LAN access points AP, network cameras, etc.) while transmitting data signals without changing any existing Ethernet Cat.5 cabling basic framework. This kind of power supply has the advantage of centralized power supply. By taking this advantage, POE power supply control terminal (POE network switch) can be connected to fire emergency power supply to solve the problem that the external power supply disconnects with WLAN when the fire occurs. The power access method can guarantee the effectiveness of WLAN over a period of time (fire emergency power system has energy supply).

\section{Firefighter positioning and monitoring system technology scheme.}

In wireless communication network, the basic principle of wireless positioning is to measure some parameters of radio waves and determine the position of measured wireless terminal according to specific algorithm. Measurement parameters generally include the propagation time, amplitude, phase and angle of arrival of radio waves. The specific positioning accuracy depends on the measurement method. From the basic principle of positioning, it can be roughly divided into the following three types: positioning methods based on signal measurement operation, on scene analysis and on proximity relation.

Positioning method based on signal measurement operation. This positioning method uses geometrical trigonometric or hyperbolic relationship to calculate the position of measured object based on the propagation time, amplitude, phase and angle of arrival of the acquired radio waves. It is the most important and most widely used positioning technology. This positioning method is more suitable for wireless positioning in outdoor open spaces. When wireless positioning is performed in a building, the signal attenuation is unknown because the signal needs to penetrate or diffract obstacles; at the same time, different building materials have different signal absorption rates. Therefore, the accuracy of the positioning in a building is related to many factors, and the positioning accuracy is difficult to guarantee. In particular, a positioning method based on angle measurement needs a directional antenna, such as smart antenna array. Therefore, this positioning method is not suitable for WLAN wireless positioning in a building.

Positioning method based on scene analysis. The positioning method based on scene analysis refers to the abstraction and formalization of specific environment that need to be positioned, describing specific positions in the environment with some specific and quantitative parameters and integrating these information together in a database. The observer queries the database according to the "fingerprint" feature of the position of the wireless terminal to be positioned and determines the position of the object according to specific matching rule. Prior to the implementation of the positioning method, it is necessary to collect "fingerprint" information for specific environment to form a positioning parameter database. The accuracy of the positioning parameter database is closely related to the actual positioning accuracy and the workload of the previous period will be very large.

Positioning method based on proximity relation. The principle of the positioning method based on proximity relation is to position the object based on the proximity relation between the object to be positioned and one or more known position reference points. This positioning technique usually requires the aid of the unique identification system to determine the known positions. The most common example is Cell ID in mobile cellular communications network. It is assumed that the objects to be positioned are in three cells respectively. Since the position of the reference point in each Cell is known, the position of the reference point in Cell can be roughly determined according to Cell which is the position of the reference point in Cell. Besides Cell, other examples are Xerox PareTAB System, Carnegie Mellon Andrew, Active Badge, etc.

The positioning method applied in this paper is based on proximity relation. Since the object to be positioned (wireless access terminal) needs to access WLAN through a certain AP in the network and has uniqueness, according to the correspondence between AP and the floor plan of the building, 
the actual position of the object to be positioned can be obtained. The accuracy of this positioning method depends on the density of APs within the building. If the coverage of AP is small, the positioning accuracy will be very high; conversely, if AP coverage is large, the positioning accuracy will be low. Taking hotel as an example. At present, each room is covered by one AP. This positioning method can position one person's floor and room number at a certain moment, which can provide valuable rescue time for rescue personnel or firefighters of public security firefighting force.

\section{Firefighter Positioning and Monitoring System}

\section{System Components .}

A wireless network firefighter positioning and monitoring system based on thin AP framework consists of multiple ACs (radio access controllers), multiple POE switches, multiple APs (wireless access points), multiple ATs (radio access terminals), multiple positioning clients and one positioning server. The framework of the wireless network firefighter positioning and monitoring system based on thin AP framework is as follows:

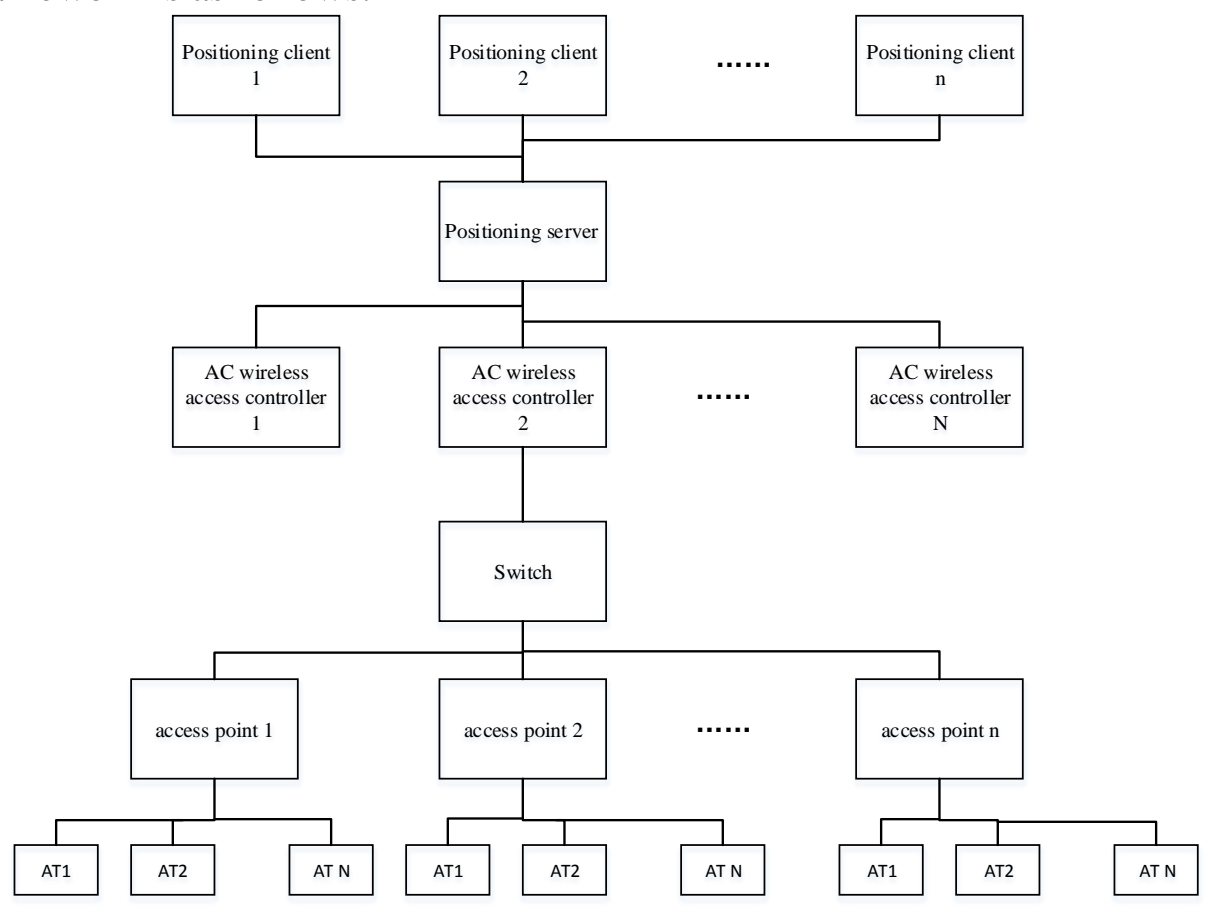

Fig 1 Structure of a wireless network firefighter positioning and monitoring system based on thin AP framework

In the figure above, the power of the wireless access controller and the switch (which can be multiple switches and can be cascaded to multiple APs) is powered through the fire emergency power system, ensuring the normal work when the external power supply of the building is disconnected. The connection between wireless access controller, switch and wireless access point is through a network cable, and the positioning server and the positioning client can access WLAN of a building through wired or wireless access. The wireless access terminals are connected by both wireless and wired ways.

\section{System functions.}

The main purpose of the wireless network firefighter positioning and monitoring system based on thin AP framework is to make use of WLAN network inside buildings to realize the positioning of the trapped people and the firefighters entering buildings to carry out rescue and firefighting in order to give play to the role of personnel positioning, search and rescue and security when a sudden event or fire occurs in hotels, restaurants and large entertainment places. 
The function of the positioning server. The positioning server establishes a real-time connection with radio access controller in uplink and periodically receives the access routing table of the radio access terminal from the radio access controller, and stores the routing table on the server, and establishes a real-time connection with the positioning client; The positioning server establishes a real-time connection with the positioning client in downlink, receives the positioning application from the positioning client and returns results to the positioning client.

The positioning client. The main function of the positioning client is to establish a real-time connection with the positioning server, send a positioning application to the positioning server according to the ID of the person to be positioned, receive positioning results and present to the commander in an intuitive and brief manner.

AC (Wireless Access Controller). The wireless access controller establishes a real-time connection with multiple wireless access points in uplink and acquires the access information table of the wireless access terminal according to a certain time interval, and stores the access information of the wireless access terminal on the wireless controller; The wireless access controller establishes a real-time connection with the positioning server in downlink and periodically uploads the access information of the wireless access terminal on the wireless controller to the positioning server.

POE switch. In addition to the ordinary functions, POE switch also has the following two functions. It can provide power for the wireless access point, and it can be served as data communication bridge between the wireless access controller and the wireless access point. The access power of POE switch comes from the fire emergency power system to ensure that WLAN inside the building can also work normally when the external power supply of the building is disconnected due to a sudden accident.

AP (Wireless Access Point). The wireless access point can establish a real-time connection with the wireless access controller in an uplink, periodically report the access information table of the wireless access terminal, and accept the access application of the wireless access terminal in downlink.

AT (Wireless Access Terminal). As personal terminals carried or worn by firefighters, wireless access terminals can dynamically access wireless access points according to the rules (encryption algorithms, keys).

\section{Firefighters positioning and monitoring system data transmission method}

Access information table of AT wireless access terminal on AP wireless access point

The information sheet is as follows:

\{AP_MAC address: [sequence code, AT access number n, command code, [[AT1_mac, \{AT access information]], [AT2_mac, \{AT access information $\}]$, [ATn_mac, \{AT access information $\}]$ ] ],..... ;

"Sequence code" and "command code" users can define their own contents in the positioning algorithm.

AT access information con access point 1tents are as follows:

\{AT sending baud rate, AT sending byte count, AT receiving baud rate, AT receiving byte count, AT signal field strength, AT signal mean field strength .

Data flow between the positioning client and the positioning server 


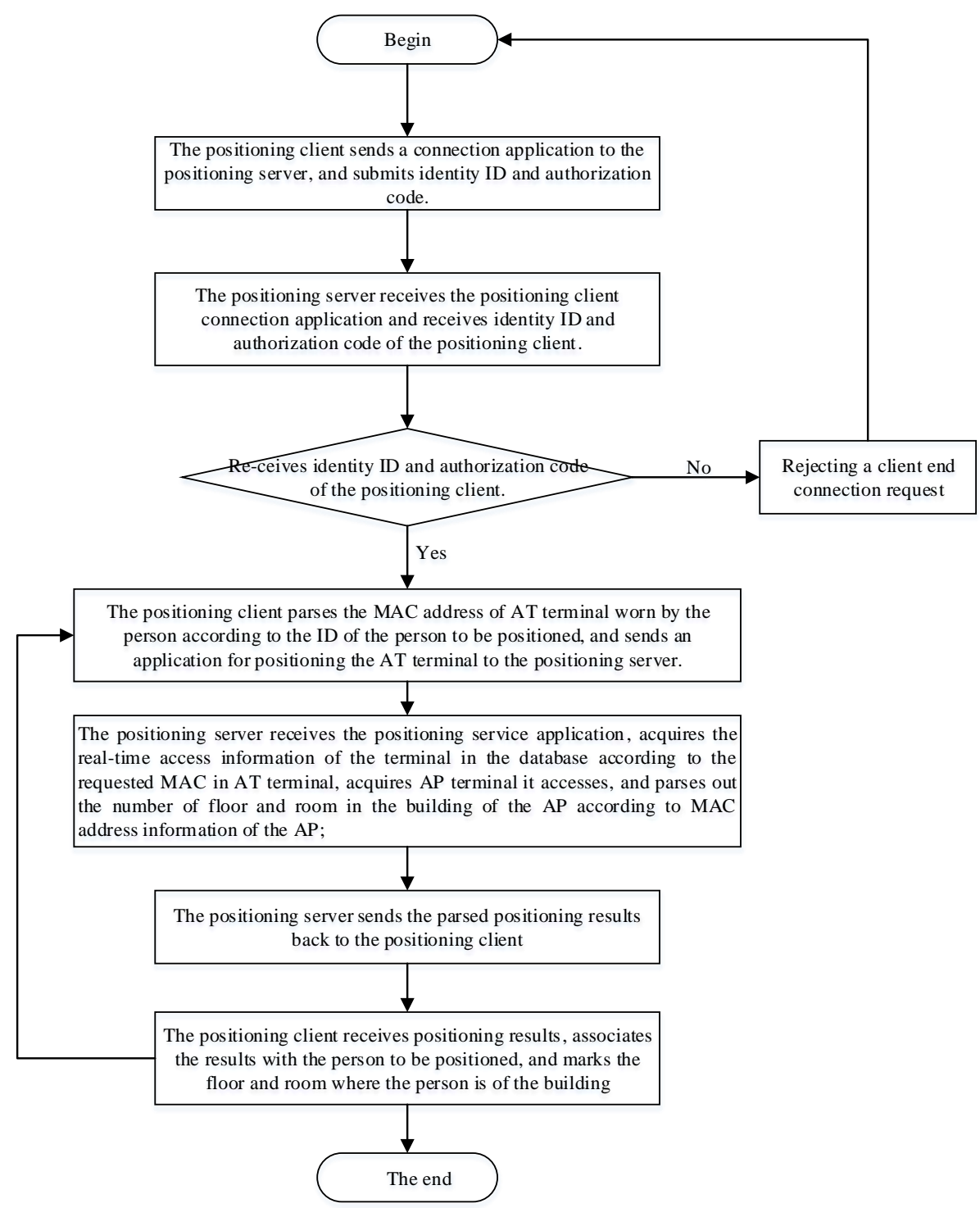

Fig 2 Data flow between the positioning client and the positioning server

The steps are as follows:

(1) Begin;

(2) The positioning client sends a connection application to the positioning server, and submits identity ID and authorization code.

(3) The positioning server receives the positioning client connection application and receives identity ID and authorization code of the positioning client.

(4) The positioning server compares identity ID and authorization code in the database to determine whether identity ID matches the authorization code, in order to prevent access by unauthorized users. If matches, turn to (5), otherwise, turn to (9).

(5) The positioning client parses the MAC address of AT terminal worn by the person according to the ID of the person to be positioned, and sends an application for positioning the AT terminal to the positioning server.

(6) The positioning server receives the positioning service application, acquires the real-time access information of the terminal in the database according to the requested MAC in AT terminal, acquires AP terminal it accesses, and parses out the number of floor and room in the building of the $\mathrm{AP}$ according to MAC address information of the AP;

(7) The positioning server sends the parsed positioning results back to the positioning client;

(8) The positioning client receives positioning results, associates the results with the person to be positioned, and marks the floor and room where the person is of the building; 
(9) If the connection application of the positioning client is denied, then turn to (2);

(10) The end.

The data flow of AC wireless access controller

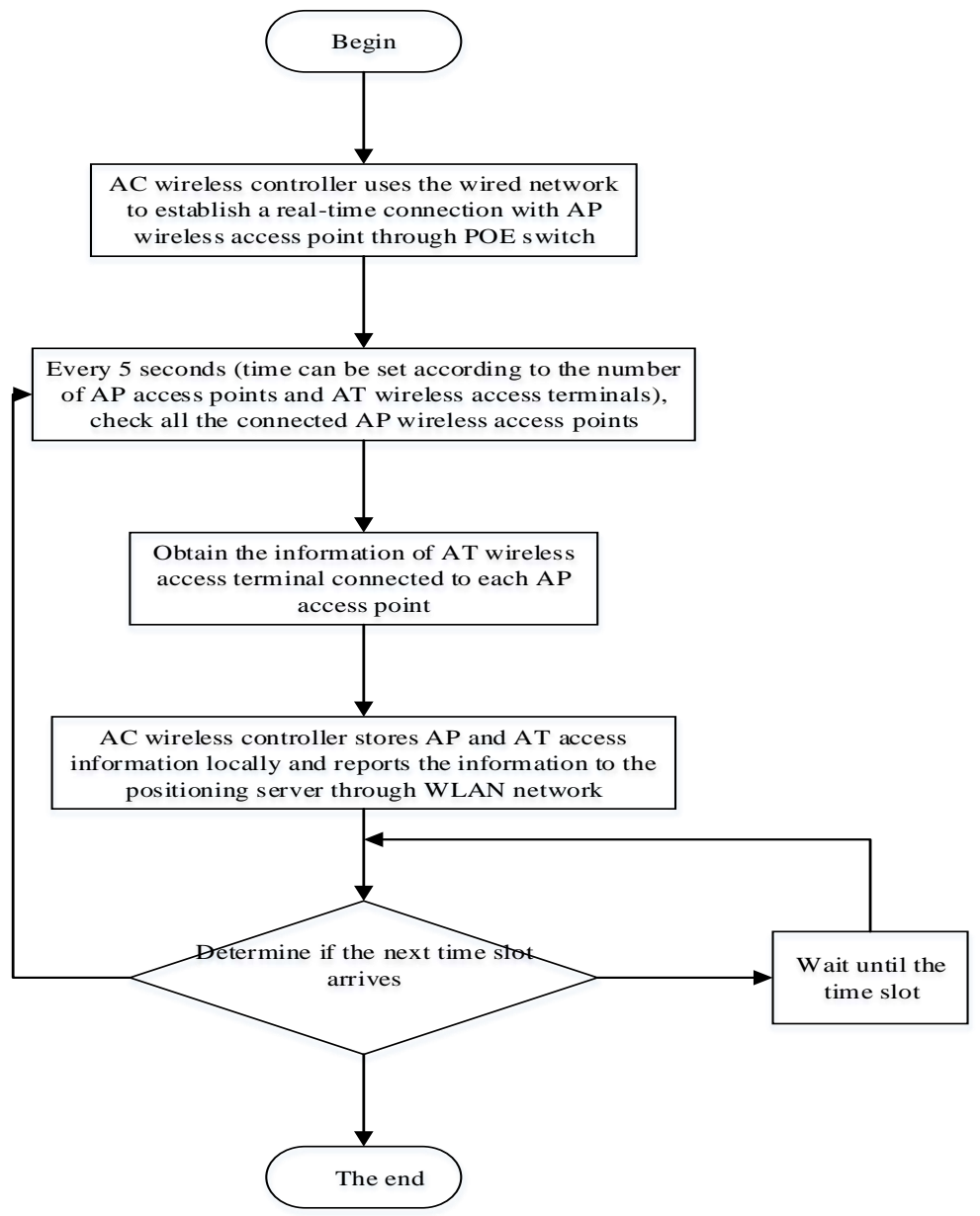

Fig 3 The data flow of $\mathrm{AC}$ wireless access controller

The steps are as follows:

(1) Begin;

(2) AC wireless controller uses the wired network to establish a real-time connection with AP wireless access point through POE switch;

(3) Every 5 seconds (time can be set according to the number of AP access points and AT wireless access terminals), check all the connected AP wireless access points;

(4) Obtain the information of AT wireless access terminal connected to each AP access point;

(5) AC wireless controller stores AP and AT access information locally and reports the information to the positioning server through WLAN network.

(6) Determine if the next time slot arrives, if yes, turn to (7), otherwise, turn to (10);

(7) Turn to (3);

(8) Wait until the time slot arrives, turn to (6);

(9) The end.

\section{Conclusion}

The actual test verifies that the wireless network firefighter positioning and monitoring system and data transmission method based on thin AP framework proposed in this paper can meet the actual needs of firefighting force positioning in the public security and provide a new technology means for the search and guarantee for fire fighting and rescue personnel.

The advantages of the system 
Investment is low. The positioning scheme mentioned in this paper can use all networking devices in existing WLAN network in a specific place, but it is only necessary to improve or replace $\mathrm{AC}$ access controller, and the building owner do not have to put a lot of manpower and physical costs caused by the increase of location services;

Positioning accuracy can be controlled. In the positioning method used in this paper, the accuracy of positioning depends on the coverage and number of AP wireless access points in the building. To improve the positioning accuracy of the system in a specific place, multiple APs can be installed;

Application is wide. The positioning scheme can ensure that WLAN can be applied inside buildings during fires or other unexpected events. Therefore, audio, video and other data communication services can be loaded according to actual conditions. Especially in recent years, with the emergence of personal terminal equipment, data, such as vital signs of firefighters, can be collected through the bracelet device, and the data can be uploaded to the fire mobile communication command center or other centers through WLAN network to realize the real-time monitoring of vital signs of firefighters.

\section{References}

[1] Wang Xiaorui,Xing Guoliang,Zhang Yuanfang,et al.Integrated coverage and connectivity configuration in wireless sensor networks. Proceedings of the 1st International Conference on Embedded Networked Sensor Systems. 2003.

[2] Jiang D,Delgrossi L.IEEE802.11p: towards an international standard for wireless access invehicular environments. Proceedings of67th IEEE vehicular technology conference onvehicular technology . 2008.

[3] Sylvie Delaët,Partha Sarathi Mandal,Mariusz A. Rokicki,Sébastien Tixeuil. Deterministic secure positioning in wireless sensor networks[J]. Theoretical Computer Science . 2011 (35) .

[4] Nan Li,Burcin Becerik-Gerber. Performance-based evaluation of RFID-based indoor location sensing solutions for the built environment[J]. Advanced Engineering Informatics . 2011 (3).

[5] Wu Cheng. Research on a positioning-based manufacturing execution system based on the Internet of Things[D]. University of Electronic Science and Technology of China, 2013.

[6] Du Haitao, Quan Chunlai, Zhou Xiang. Research on indoor positioning technology based on wireless sensor and video fusion[J]. Computer Engineering and Design. 2011(01).

[7] Wang Yuan, Lin Jin'guo. Discussion on several common indoor positioning technologies[J]. China Instrumentation. 2011(02). 